\title{
Internet das coisas aplicada no ambiente das bibliotecas: uma revisão sistemática da literatura internacional
}

Fernanda Vasconcelos AmaraI

http://orcid.org/0000-0001-6197-9548

Jordan Paulesky Juliani II

http://orcid.org/0000-0001-7823-6644

Raphael Winckler de Bettio ${ }^{\text {II }}$

http://orcid.org/0000-0003-3635-3580

I Universidade do Estado de Santa Catarina, SC, Brasil.

Mestre em Gestão da Informação.

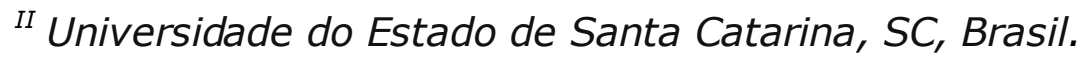
Doutor em Engenharia e Gestão do Conhecimento pela Universidade Federal de Santa Catarina. Professor do Departamento de Educação Científica e Tecnológica.

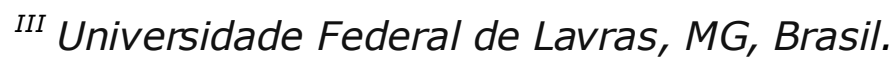

Doutor em Engenharia e Gestão do Conhecimento pela Universidade Federal de Santa Catarina. Professor do Departamento de

Ciência da Computação na Universidade Federal de Lavras.

http://dx.doi.org/10.1590/1981-5344/4048

O presente artigo analisa a produção científica internacional com a temática de aplicação de internet das coisas (IoT) em bibliotecas. Constituem-se como objetivos dessa análise: mapear a produção acadêmica sobre aplicação da tecnologia de IoT em bibliotecas desde o surgimento das primeiras discussões sobre essa tecnologia até o ano de 2018; verificar se os estudos são práticos ou apenas teóricos; identificar as áreas de pesquisa e países que mais desenvolvem estudos sobre 
essa temática; apurar as aplicações mais comuns em bibliotecas e quais tecnologias estão sendo utilizadas; identificar iniciativas práticas bem-sucedidas em bibliotecas. A metodologia adotada é a revisão sistemática da literatura internacional disponível nas bases de dados Scopus e Web of Science. O foco desse levantamento bibliográfico é identificar pesquisas sobre IoT exclusivamente no ambiente das bibliotecas. Trata-se uma pesquisa exploratória com a finalidade de caracterizar a produção científica sobre essa temática e a análise dos dados segue uma abordagem quantiqualitativa. Conclui-se que as pesquisas de IoT em biblioteca ainda são escassas, com predominância de estudos teóricos em detrimento de experimentos empíricos. A área de Ciência da Computação concentra o maior número de publicações e pode ser beneficiada ao realizar uma parceria interdisciplinar com a Biblioteconomia e Ciência da Informação.

Palavras-chave: internet das coisas; bibliotecas; revisão sistemática; inovação em bibliotecas; bibliotecas inteligentes.

\section{falta título inglês}

This article analyzes the international scientific production with the theme of Internet application of things (IOT) in libraries. The objectives of this analysis are: map the academic production on the application of IoT technology in libraries since the first discussions about this technology until the year 2018; verify whether the studies are practical or only theoretical; identify the research areas and countries that develop the most studies on this subject; to determine the most common applications in libraries and which technologies are being used; identify successful practical initiatives in libraries. The methodology adopted is the systematic review of international literature available in the Scopus and Web of Science databases. The focus of this bibliographic survey is to identify research on IoT exclusively in the library environment. It is an exploratory research with the 
purpose of characterizing the scientific production on this subject and the data analysis follows a quanti-qualitative approach. It is concluded that the research of IoT in library is still scarce, with predominance of theoretical studies to the detriment of empirical experiments. The area of Computer Science concentrates the largest number of publications and can benefit from an interdisciplinary partnership with Librarianship and Information Science.

Keywords: internet of things; libraries; systematic review; innovation in libraries; smart libraries.

Recebido em 16.07.2019 Aceito em 20.10.2020

\section{Introdução}

O aumento da inserção de tecnologias no cotidiano da população trouxe inúmeras comodidades e facilidades, além de agilizar o desenvolvimento de tarefas e a obtenção de informações tanto na esfera pessoal quanto profissional. Atividades que anteriormente eram complicadas e demoradas, como determinar a rota para se dirigir a um local desconhecido, atualmente são facilitadas por recursos tecnológicos como o GPS, por exemplo. No ambiente de trabalho, relatórios com dados estatísticos, gráficos e tabelas podem ser gerados automaticamente, sem a necessidade de um profissional coletar e estruturar esses dados manualmente, de forma laboriosa e morosa.

O ambiente das bibliotecas não ficou imune a esses avanços tecnológicos,com a introdução de sistemas de gerenciamento de acervo, bases de dados, máquinas de auto empréstimo, entre outros recursos. Dessa forma, profundas mudanças ocorreram nas rotinas realizadas nesses espaços: as fichas catalográficas foram substituídas por catálogos de acesso on-line, os empréstimos de livros registrados em fichas de papel são realizados atualmente em sistemas informatizados, o Portal de Periódicos da Capes sucedeu as bases de dados em CD-ROM.

A incorporação das novas tecnologias possibilitou diversificar e melhorar os serviços e produtos ofertados pelas bibliotecas. Além disso, as tarefas rotineiras foram automatizadas, gerando economia de custo e tempo empregados na execução dos processos de trabalhos dessas unidades de informação. Contudo, a medida que surgem novas 
ferramentas e recursos tecnológicos, é responsabilidade dos bibliotecários verificar os possíveis aprimoramentos que podem ser introduzidos para continuar a multiplicar a eficiência no atendimento às necessidades dos usuários.

Atualmente, entre diversas tecnologias emergentes com grande potencial de aplicabilidade em unidades de informação, destaca-se a internet das coisas (IoT). Com o objetivo de conectar qualquer objeto físico a internet, a IOT permite que objetos analógicos como geladeiras, carros ou livros coletem e transmitam dados por meio de sensores inteligentes neles instalados. Dessa forma, será possível, por exemplo, que a sua geladeira avise quando um produto em seu interior está com a data de validade vencida e precisa ser jogado fora, ou a estante de uma biblioteca informe quando um livro é guardado no local errado.

Ao interligar objetos a internet, a IoT aumentará exponencialmente a quantidade de dados e informações capturados, processados e transmitidos, tudo em tempo real. Dentro dos domicílios, a IoT poderá monitorar o ambiente e ajustar a temperatura e luminosidade da casa automaticamente de acordo com as preferências do morador. No setor de logística e transporte, cargas serão rastreadas para garantir sua segurança e correta destinação. Na área da saúde, tratamentos médicos serão capazes propiciar menos efeitos colaterais ao ter sistemas que monitorem o corpo humano de forma muito mais precisa.

Com tanta versatilidade, o emprego da internet das coisas é providencial para potencializar a utilização das bibliotecas e oferecer serviços cada vez mais personalizados. A IoT possui diversas funcionalidades com capacidade para tornar o ambiente físico da biblioteca mais atraente e moderno, fidelizando os usuários atuais e conquistando um novo público interessado em frequentar o local.

Serviços de recomendações, rastreamento de livros, monitoramento automático do ambiente físico em relação a temperatura e luminosidade, facilidades para acesso e locomoção dentro da biblioteca, entre outros serviços, criará uma biblioteca altamente eficiente em atender o público. A oferta dessas novidades nas bibliotecas é imprescindível para manter a relevância desses espaços, que sofrem uma disputa acirrada com outros ambientes de informação, em especial a internet. A IOT ao possibilitar que os dados dos usuários, dos materiais bibliográficos disponibilizados e dos caminhos percorridos dentro da biblioteca sejam utilizados, possibilitará a biblioteca oferecer serviços customizados, na medida da demanda de seus usuários.

Por isso, no presente artigo é proposto analisar publicações científicas sobre aplicação de IoT em bibliotecas, disponíveis nas bases de dados Scopus e Web of Science. O objetivo é identificar o panorama das 
pesquisas desenvolvidas sobre essa tecnologia no ambiente das bibliotecas para compreender a sua evolução.

Para contextualizar o problema de pesquisa, primeiramente, é apresentado uma revisão de literatura sobre o conceito de IoT, suas funcionalidades e aplicações. Essa seção discorre sobre conceitos correlacionados, como a computação pervasiva e ubíqua, e identifica a aplicação de IoT em diferentes ambientes, com destaque para as bibliotecas. Esse panorama teórico é essencial para entender o significado do termo internet das coisas, que ainda é um conceito nebuloso, devido seu recente surgimento e complexidade. A seção de metodologia discorre sobre a estratégia de busca utilizada na revisão sistemática e os critérios de exclusão de publicações. Em resultados e discussão são analisados os dados estatísticos, tabelas e gráficos sobre as publicações localizadas. Por fim, apresenta-se a síntese dos resultados encontrados e as considerações finais.

\section{IoT: definição, funcionalidades e aplicações}

Em uma busca incansável pelo aprimoramento tecnológico, novos produtos são desenvolvidos diariamente, com um nível de sofisticação crescente. Computadores e celulares, por exemplo, diminuíram de tamanho e aumentaram sua capacidade de processamento de dados. Dessa forma, é possível que esses novos inventos se mesclem mais organicamente ao ambiente analógico e estejam disponíveis para uso em variados lugares e momentos. A proposta da computação pervasiva e ubíqua é justamente permitir que a tecnologia seja onipresente no cotidiano da sociedade, auxiliando os indivíduos de forma ininterrupta, em qualquer lugar, a qualquer hora (DOHR et. al, 2010).

Nesse contexto de evolução tecnológica, Ashton (2009) discorre que o surgimento da internet propiciou, primeiramente, que computadores fossem conectados em rede. Em seguida, as pessoas foram conectadas por meio de recursos como e-mails, mensagens instantâneas, fóruns, seção de comentários e, mais recentemente, por meio das redes sociais. Com a IoT, objetos e ambientes físicos serão conectados em rede e terão suas funcionalidades ampliadas por meio da coleta, transmissão e cruzamentos de dados. Dessa forma, o mundo físico estará conectado quase na sua totalidade com o mundo digital, o que trará informações em tempo real, conforto, produtividade e praticidade para a sociedade.

O termo internet das coisas foi mencionado pela primeira vez em 1999 por Kevin Ashton, um pesquisador britânico do Massachusetts Institute of Technology, durante um evento sobre RFID, a tecnologia de identificação por rádio frequência (ASHTON, 2009). Simplificadamente, a IoT conecta objetos físicos à internet para gerar produtos e serviços com 
alto valor agregado e sem a necessidade inerente e permanente da intervenção humana.É importante destacar que o conceito de objeto físico deve ser entendido de uma forma bastante ampla. Esse objeto ou "coisa" na IoT pode ser tanto artefatos criados pelo homem quanto uma pessoa ou outro animal com um biochip ou um implante de monitoramento cardíaco, por exemplo (SHIN, 2010).

\section{Ashton (2009, tradução nossa) afirma que}

Hoje, os computadores - e, portanto, a Internet - são quase totalmente dependentes dos seres humanos para obter informações. Quase todos os cerca de 50 petabytes (um petabyte é 1.024 terabytes) de dados disponíveis na Internet foram capturados e criados por seres humanos - digitando, pressionando um botão de gravação, tirando uma foto digital ou digitalizando um código de barras. Os diagramas convencionais da Internet incluem servidores e roteadores e assim por diante, mas eles deixam de fora os roteadores mais numerosos e importantes de todos: as pessoas. 0 problema é que as pessoas têm tempo, atenção e precisão limitados - o que significa que eles não são muito bons em capturar dados sobre as coisas no mundo real.

$E$ isso é um grande problema. Somos físicos e nosso ambiente também. Nossa economia, sociedade e sobrevivência não são baseadas em ideias ou informações - elas são baseadas em coisas. (...). Ideias e informações são importantes, mas as coisas importam muito mais. No entanto, a tecnologia da informação de hoje é tão dependente de dados originados por pessoas que nossos computadores sabem mais sobre ideias do que sobre coisas. (...). Precisamos capacitar os computadores com seus próprios meios de coletar informações, para que possam ver, ouvir e cheirar o mundo por si mesmos, em toda a sua glória aleatória. 1

Ou seja, enquanto nos primórdios da internet os dados eram fornecidos por humanos, com a IoT esses dados serão coletados e

\footnotetext{
1 Today computers - and, therefore, the Internet - are almost wholly dependent on human beings for information. Nearly all of the roughly 50 petabytes (a petabyte is 1,024 terabytes) of data available on the Internet were first captured and created by human beings-by typing, pressing a record button, taking a digital picture or scanning a bar code. Conventional diagrams of the Internet include servers and routers and so on, but they leave out the most numerous and important routers of all: people. The problem is, people have limited time, attention and accuracy - all of which means they are not very good at capturing data about things in the real world.And that's a big deal. We're physical, and so is our environment. Our economy, society and survival aren't based on ideas or information - they're based on things. (...). Ideas and information are important, but things matter much more. Yet today's information technology is so dependent on data originated by people that our computers know more about ideas than things. (...).We need to empower co mputers with their own means of gathering information, so they can see, hear and smell the world for themselves, in all its random glory.(ASHTON, 2009).
} 
processados automaticamente por objetos com dispositivos internos para coleta e processamento de dados, denominados de objetos inteligentes. As imprecisões e inconsistências dos dados gerados, morosamente, por humanos serão eliminadas e os objetos inteligentes fornecerão informações mais fidedignas e em tempo real. Além disso, a capacidade de cruzar informações e processá-las automaticamente aumentará de forma exponencial. Por isso, a IoTestá sendo considerada por muitos analistas futurísticos como mais do que somente uma evolução da internet, seu surgimento tem potencial para revolucionar a sociedade da mesma forma que a Revolução Industrial no século XVIII e a Revolução Técnico-Cientifico-Informacional no século XX (FUNCHAL, 2017).

Há inúmeras possibilidades de aplicação de IoT nas mais diferentes áreas do conhecimento, envolvendo os segmentos da indústria, comércio e serviços. Diversos protótipos e pesquisas estão sendo desenvolvidos por empresas de tecnologia e no ambiente acadêmico, e o número de produtos e serviços com essa tecnologia deve multiplicar nos próximos anos.

Atzori, Iera e Morabito (2010) identificam quatro áreas onde a IoT deve florescer nos próximos anos:

- transporte e logística: carros, trens, ônibus e até mesmo bicicletas estão recebendo sensores, atuadores (componente que produz movimento ao receber comandados manuais, elétricos ou mecânicos) e sistemas de processamento para monitorar e controlar seu deslocamento. Rodovias e produtos transportados também recebem sensores e etiquetas para emitir e transmitir dados. Algumas aplicações práticas são o monitoramento de mercadorias transportadas; o auxílio na condução de veículos, podendo até mesmo, impedir colisões e outros acidentes; a transmissão de informações em tempo real sobre as condições de tráfego e as melhores rotas e meios de transporte a serem utilizados;

- saúde: rastreamento de objetos e pessoas; monitoramento das condições físicas de pacientes para diagnóstico e tratamento por meio de sensores que podem ser inseridos interna ou externamente no corpo do indivíduo; coleta e 
transferência automática de dados para agilizar os procedimentos dentro dos hospitais;

- ambientes inteligentes: transformação de espaços como escritórios e moradias em locais mais funcionais e confortáveis por meio de dispositivos que, por exemplo, ajustam a temperatura e luz de acordo com as preferências requisitadas ou desliga equipamentos que não estão sendo utilizados. Esses ambientes também irão monitorar as atividades que acontecem em seu interior e podem, por exemplo, alertar que um indivíduo em uma academia não está realizando suas atividades físicas na intensidade determinada em seu programa de treinamento.

- domínio pessoal e social: transmissão de informação automaticamente para outras pessoas sobre o deslocamento de um indivíduo (por exemplo informar se os filhos já chegaram na escola ou universidade); rastreamento de objetos perdidos ou roubados; consultas do histórico de objetos e datas de eventos para verificar onde, como e com quem uma pessoa gastou seu tempo em determinado período de tempo.

No ambiente das bibliotecas, existem várias possibilidades de aplicação de IoT, relacionadas tanto a promoção de facilidades na utilização dos serviços e espaços físicos disponibilizados, quanto a dinamização do acervo, por meio da criação de serviços de alerta e de recomendação, que promovam um aumento no número de itens do acervo consultados ou emprestados. Algumas das principais aplicações são (GUPTA; SINGH, 2018; HAHN, 2017; PUJAR; SATYANARAYANA, 2015):

- monitoramento do ambiente: controle automático de luz, temperatura e nível de barulho; indicação de disponibilidade de salas e equipamentos para uso; indicação dos horários de 
pico de movimento de usuários e quais espaços estão sendo mais utilizados;

- acesso à biblioteca e seus recursos: registro de usuários e permissão de entrada dentro da biblioteca por meio de um cartão virtual; pagamento de multa por celular ou outro dispositivo portátil;

- serviços de localização: criação de mapas internos do edifício da biblioteca (indoor map); localização em tempo real de usuários e dos materiais bibliográficos disponibilizados;

- serviços de recomendação: recomendação de informação baseado na localização do usuário dentro da biblioteca e no histórico de empréstimo; promoção de informações adicionais sobre um livro por meio das indicações de websites por exemplo;

- linked data: vincular as informações e 0 acervo disponibilizados pelas bibliotecas com dados da internet e aplicativos em nuvem;

- serviços de alerta: informar ao usuário em tempo real sobre livro reservado devolvido a biblioteca; multa por atraso de livro; livro novo recém disponibilizado nas estantes;

- gestão de coleções: controle de inventário; localização de materiais extraviados nas estantes; alerta sobre materiais recentemente adquiridos;

- capacitação de usuários: realidade aumentada e gamificação em treinamentos de usuários; aprendizagem baseada em objetos; tecnologia assistiva.

Dessa forma, sistemas baseados em IoT podem ser aplicados para melhorar diversos serviços oferecidos pelas bibliotecas. Essas aplicações nas bibliotecas encontram-se em variados estágios de desenvolvimento: há pesquisas sobre dispositivos e sistemas que podem ser facilmente construídos com a tecnologia atual, até estudos futuristas que preveem serviços e produtos ainda inalcançáveis. 


\section{Metodologia}

O presente estudo caracteriza-se como uma pesquisa exploratória, pois objetiva proporcionar maior familiaridade com o tema Internet das Coisas em bibliotecas. Em relação a sua finalidade, é uma pesquisa descritiva, com o propósito de analisar as publicações científicas produzidas sobre a aplicação dessa tecnologia em bibliotecas. Na análise dos dados coletados, utilizou-se uma abordagem quantitativa e qualitativa para melhor descrever e interpretar as variáveis pesquisadas.

O método adotado para coleta de dados é a revisão sistemática em bases de dados internacionais. Recorrente na área médica, esse tipo de revisão tem como finalidade localizar estudos semelhantes, avaliá-los criticamente e gerar uma análise estatística (metanálise) que sintetize as informações encontradas (SAMPAIO, MANCINI, 2007). A revisão sistemática requer a criação antecipada de uma estratégia de busca bem delineada e a determinação de critérios explícitos de inclusão e exclusão de publicações.

O procedimento metodológico envolveu as seguintes etapas:

Escolha das bases de dados: as bases Scopus e Web of Science foram escolhidas por serem dois dos maiores bancos de dados de resumos e citações da literatura revisada por pares. A pesquisa foi realizada em de abril de 2019.

Definição da estratégia de busca: dois conjuntos de palavraschaves foram definidos (QUADRO 1), um indicando a tecnologia IoT e outro especificando o local de aplicação dessa tecnologia. Não houve delimitação do ano de publicação, em virtude de ser um tema muito recente.

Quadro 1 - Estratégia de busca da revisão sistemática

\begin{tabular}{|c|c|}
\hline CONJUNTO & PALAVRAS-CHAVES \\
\hline Tecnologia & $\begin{array}{l}\text { "internet of things" } O R \text { "IOT" } O R \text { "ubiquitous computing" } O R \\
\text { "pervasive computing"OR "smart library" } O R \text { "intelligent library" }\end{array}$ \\
\hline \multicolumn{2}{|r|}{ AND } \\
\hline Local & $\begin{array}{l}\text { "academic library" } O R \text { "school library" } O R \text { "public library" } O R \\
\text { "special library" } O R \text { "university library" }\end{array}$ \\
\hline
\end{tabular}

Fonte: Do autor (2019)

O primeiro grupo de palavras-chaves consiste em sinônimos e termos correlacionados a IoT. Nota-se que muitas aplicações de IoT são enquadradas em artigos indexados como computação ubíqua/pervasiva ou tecnologia de ambiente inteligente. 
O segundo grupo identifica o ambiente de aplicação de IoT a ser estudado. Verificou-se em um pré-teste inicial que sem especificar o tipo de biblioteca, a taxa de revocação era muito alta, com uma precisão bastante reduzida, devido a polissemia dos termos "library" e "smart library", que podem referir-se a um software ou banco de dados. Por isso, optou-se por especificar os tipos de bibliotecas existentes (acadêmica/universitária, escolar, pública, especial). Apesar de não ser um termo de vocabulário controlado, a palavra-chave "university library" também recuperou alguns resultados adicionais ao termo "academic library".

Definição dos critérios de exclusão: esses critérios objetivam refinar o resultado de busca e obter apenas publicações relevantes para a pesquisa. Os seguintes padrões para exclusão foram definidos:

1. documentos que, por meio da análise do título e resumo, estão fora do escopo da pesquisa (predominantemente tratam do termo biblioteca enquanto um banco de dados/software);

2. documentos sobre aplicação de IoT em diversos contextos (indústrias, casas, espaços públicos, etc.) e que apenas citam brevemente a aplicação de IoT em bibliotecas, sem que o assunto seja aprofundado;

3. documentos sobre inovação em bibliotecas e que apenas citam brevemente a aplicação de IoT, sem que o assunto seja aprofundado;

4. documentos sobre o tema "smart/intelligent library" que não abordam a aplicação de soluções de IoT;

5. documentos publicados em línguas com sistemas de escrita ideográficos (chinês, coreano, japonês, etc.).

Execução da busca nas bases de dados e análise dos documentos recuperados: como resultado preliminar, foram recuperados 50 documentos na Scopus e 13 na Web of Science. Após a aplicação dos critérios de exclusão, foram obtidas 11 publicações na Scopus e 10 na Web of Science, dos quais 6 apareceram nas duas bases. Dessa forma, o resultado final da busca recuperou 15 documentos de interesse para a pesquisa. 
O critério de exclusão mais utilizado foi para documentos que tratam de inovação em bibliotecas, mas sem foco em IoT. Há várias pesquisas que buscam modernizar o espaço das bibliotecas, implementar melhorias tecnológicas e criar ambientes inteligentes por meio do uso do RFID principalmente, mas as inovações indicadas são bem menos sofisticadas do que o conceito de internet das coisas.

O critério 2, documentos sobre IoT que apenas citam a biblioteca, também foi bastante utilizado. As bibliotecas e museus são considerados por grande parte dos autores como locais extremamente propícios para implementar internet das coisas e, por isso, são quase sempre mencionados como ambientes com grande tendência para serem modernizados e melhorados com a IoT.

\section{Resultados e discussão}

Ao todo, quinze documentos foram recuperados sobre aplicação de internet das coisas em bibliotecas (TABELA 1). O número reduzido de material bibliográfico encontrado demonstra a incipiência da aplicação de IoT no ambiente das bibliotecas e a necessidade do desenvolvimento de pesquisas voltadas ao tema.

Tabela 1 - Visão geral dos trabalhos selecionados

\begin{tabular}{|c|c|c|c|c|c|}
\hline CITAÇÃO & ANO & $\begin{array}{c}\text { TIPO DE } \\
\text { PESQUIS A }\end{array}$ & APLICAÇÃO & TECNOLOGIA & PAÍS \\
\hline Gupta e Singh & 2018 & $\begin{array}{l}\text { Pesquisa } \\
\text { básica/teórica }\end{array}$ & $\begin{array}{l}\text { Tour virtual para orientar } \\
\text { usuários; orientação pelas } \\
\text { estantes de acordo com a lista de } \\
\text { preferência do usuário; } \\
\text { pagamento de mu lta on-line; } \\
\text { indicação da disponibilidade de } \\
\text { máquinas e recursos na } \\
\text { biblioteca; serviços de alerta. } \\
\end{array}$ & $\begin{array}{l}\text { RFID; siste ma de } \\
\text { comunicação sem fio } \\
\text { (wireless); sensores; } \\
\text { tecnologias de coleta de } \\
\text { energia; computação em } \\
\text { nuvem; protocolo de } \\
\text { internet avançada (IPv6); } \\
\text { tecnologia Magic Mirror. } \\
\end{array}$ & Índia \\
\hline Cao, Liang e Li & 2018 & $\begin{array}{l}\text { Pesquisa } \\
\text { básica/teórica }\end{array}$ & $\begin{array}{l}\text { Integração de tecnologia, } \\
\text { serviços e aprendizagem hu mana } \\
\text { na criação de bibliotecas } \\
\text { inteligentes. }\end{array}$ & $\begin{array}{l}\text { WAP (Wire less Application } \\
\text { Protocol), QR code, RSS, } \\
\text { GPS, RFID, iBeacons, } \\
\text { sensores. }\end{array}$ & China \\
\hline Liang & 2018 & $\begin{array}{l}\text { Pesquisa } \\
\text { básica/teórica }\end{array}$ & $\begin{array}{l}\text { Biblioteca } 4.0 \text {, serviços e gestão } \\
\text { do acervo inteligentes. }\end{array}$ & RFID, rede WSN. & China \\
\hline Liang e Chen & 2018 & $\begin{array}{l}\text { Pesquisa } \\
\text { básica/teórica }\end{array}$ & $\begin{array}{l}\text { Gestão de acervo e serviços } \\
\text { inteligentes. }\end{array}$ & $\begin{array}{l}\text { Sistema ciberfísico (CPS), } \\
\text { computação em nuvem, } \\
\text { Magic Mirror, sensores, } \\
\text { WSN, RFID. } \\
\end{array}$ & China \\
\hline $\begin{array}{l}\text { Grossmann, Illig } \\
\text { e Matejka }\end{array}$ & 2017 & $\begin{array}{l}\text { Pesquisa } \\
\text { aplicada/empírica } \\
\text { (Avaliação de } \\
\text { protipo na } \\
\text { University } \\
\text { Library of } \\
\text { Bamberg, } \\
\text { Alemanha) }\end{array}$ & $\begin{array}{l}\text { Monitoramento inteligente de } \\
\text { temperatura e hu midade da } \\
\text { biblioteca. }\end{array}$ & $\begin{array}{l}\text { Computador de placa única; } \\
\text { Docker e Docker swarm } \\
\text { (ferramenta de criação de } \\
\text { cluster); sensores DHT22; } \\
\text { interfaces GPIO ou USB; } \\
\text { receptores USB-WDE1; } \\
\text { dispositivos de sensores } \\
\text { autónomos ASH22; infra- }\end{array}$ & Alemanha \\
\hline
\end{tabular}


Internet das coisas aplicada no ambiente das bibliotecas: uma revisão sistemática da literatura internacional
Fernanda Vasconcelos Amaral; Jordan Paulesky Juliani; Raphael Winckler de Bettio

\begin{tabular}{|c|c|c|c|c|c|}
\hline & & & & $\begin{array}{l}\text { estrutura de rede LAN e } \\
\text { WLAN. }\end{array}$ & \\
\hline Nie & 2016 & $\begin{array}{l}\text { Pesquisa } \\
\text { básica/teórica }\end{array}$ & $\begin{array}{l}\text { Monitoramento inteligente do } \\
\text { ambiente; gestão de acervo } \\
\text { (rastreamento, empréstimo e } \\
\text { devolução automáticos de } \\
\text { liv ros); serviços personalizados e } \\
\text { precisos. }\end{array}$ & RFID. & China \\
\hline Sun e Gol & 2016 & $\begin{array}{l}\text { Pesquisa } \\
\text { aplicada/empírica }\end{array}$ & $\begin{array}{l}\text { Sistema para acelerar processo } \\
\text { de busca de livros e periódicos } \\
\text { nas estantes. }\end{array}$ & $\begin{array}{l}\text { RFID; método IOT-LM, } \\
\text { Manchester code system. }\end{array}$ & China \\
\hline Wójcik & 2016 & $\begin{array}{l}\text { Pesquisa } \\
\text { básica/teórica }\end{array}$ & $\begin{array}{l}\text { Gestão de acervo; Serviço de } \\
\text { Referência; desenvolvimento de } \\
\text { treinamentos e eventos; serviços } \\
\text { de rastreamento; marketing e } \\
\text { divulgação. } \\
\end{array}$ & RFID, Beacons, sensores. & Polonia \\
\hline $\begin{array}{l}\text { GALVÃO, J. R. } \\
\text { et al. }\end{array}$ & 2015 & $\begin{array}{l}\text { Pesquisa } \\
\text { aplicada/emp írica } \\
\text { (Biblioteca } \\
\text { Pública José } \\
\text { Saramago) } \\
\end{array}$ & $\begin{array}{l}\text { Monitoramento inteligente de } \\
\text { temperatura e eletricidade da } \\
\text { biblioteca. }\end{array}$ & $\begin{array}{l}\text { Painéis fotovoltaicos (PV); } \\
\text { caldeiras de bio massa. }\end{array}$ & Portugal \\
\hline $\begin{array}{l}\text { Polycarpou, } \\
\text { Samaras e } \\
\text { Sahalos }\end{array}$ & 2014 & $\begin{array}{l}\text { Pesquisa } \\
\text { aplicada/emp írica } \\
\text { (Projeto piloto } \\
\text { para biblioteca } \\
\text { universitária) }\end{array}$ & $\begin{array}{l}\text { Sistema de gerenciamento de } \\
\text { biblioteca com inventário, } \\
\text { localização e devolução de livros } \\
\text { de forma inteligente. }\end{array}$ & RFID & Chipre/Grecia \\
\hline $\mathrm{Li}$ & 2014 & $\begin{array}{l}\text { Pesquisa } \\
\text { básica/teórica }\end{array}$ & $\begin{array}{l}\text { Autoemprésitmo e } \\
\text { autodevolução; inventário } \\
\text { inteligente; segurança do acervo; } \\
\text { serviços personalizados. } \\
\end{array}$ & RFID & China \\
\hline Wang e Katabi & 2013 & $\begin{array}{l}\text { Pesquisa } \\
\text { aplicada/emp írica } \\
\text { (Massachusetts } \\
\text { Institute of } \\
\text { Technology) } \\
\end{array}$ & $\begin{array}{l}\text { Localização de livros nas } \\
\text { estantes. }\end{array}$ & $\begin{array}{l}\text { RFID; synthetic aperture } \\
\text { radar (SAR); dynamic time } \\
\text { warping (DTW). }\end{array}$ & EUA \\
\hline Hui & 2013 & $\begin{array}{l}\text { Pesquisa } \\
\text { básica/teórica }\end{array}$ & $\begin{array}{l}\text { Serviços personalizados baseada } \\
\text { em mineração de dados e IoT. }\end{array}$ & $\begin{array}{l}\text { Não cita nenhuma } \\
\text { tecnologia. }\end{array}$ & China \\
\hline Markakis et al. & 2013 & $\begin{array}{l}\text { Pesquisa } \\
\text { aplicada/empírica }\end{array}$ & Estantes inteligentes. & $\begin{array}{l}\text { RFID, Specific Absorption } \\
\text { Rate (SAR). } \\
\end{array}$ & Chipre/Grecia \\
\hline Luo, Cao e Qian & 2013 & $\begin{array}{l}\text { Pesquisa } \\
\text { básica/teórica }\end{array}$ & $\begin{array}{l}\text { Criação de bibliotecas } \\
\text { inteligentes. }\end{array}$ & $\begin{array}{l}\text { RFID, computação nas } \\
\text { nuvens, processadores de } \\
\text { informação em larga escala } \\
\end{array}$ & China \\
\hline
\end{tabular}

Fonte: Do autor (2019)

Os artigos mais antigos datam do ano de 2013, confirmando o surgimento recente das pesquisas sobre IoT em bibliotecas (GRÁFICO 1). Destaca-se uma queda do número de publicações sobre o tema nos anos de 2014, 2015 e 2017. Em 2016, houve um retorno no interesse sobre o 
assunto, que se manteve no ano de 2018, quando o número de publicações voltou a crescer.

Gráfico 1 - Evolução da quantidade de publicações por ano

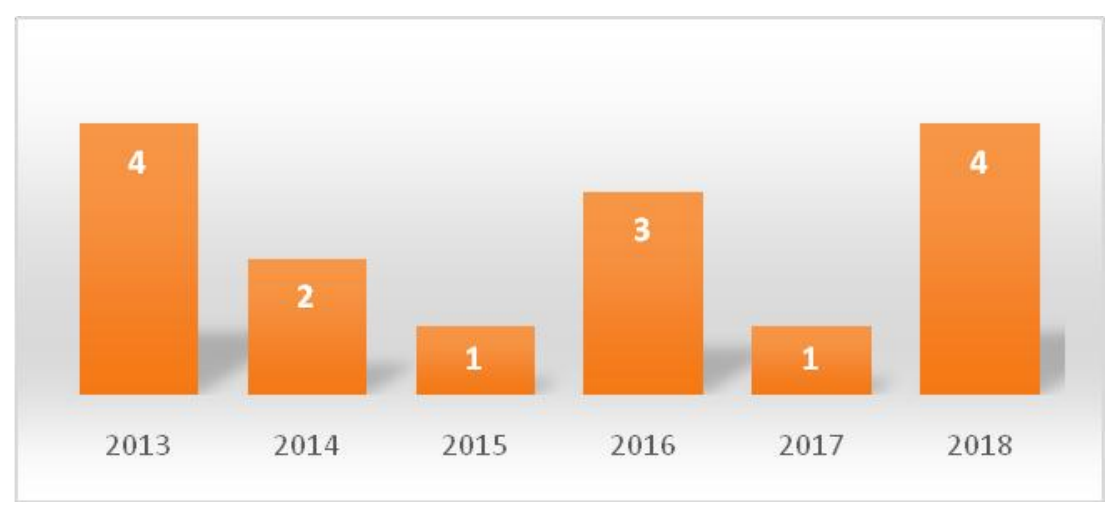

Fonte: Do autor (2019)

Embora difícil de justificar o motivo do declínio do número de artigos, nota-se na área tecnológica a emergência, de tempos em tempos, de um boom de interesse em torno de alguma nova tecnologia, que, após superada a fase de euforia com a novidade, pode ser seguida por uma certa estagnação. Isso ocorreu, por exemplo, nos $2000 \mathrm{com}$ a bolha da internet ou bolha das "empresas ponto com", quando a euforia do mercado com a criação de produtos e serviços on-line foi substituída por descrença na capacidade dessas empresas se manterem ativas a longo prazo. Dessa forma, é comum que ocorra um excesso de entusiasmo com novidades tecnológicas, seguida por um certo ceticismo em relação a cenários tão otimistas. Até que, finalmente, haja um equilíbrio entre as projeções futurísticas e a prática da implementação de novas tecnologias de forma permanente e estável. O ressurgimento de pesquisas sobre IoT em bibliotecas nos anos de 2016 e 2018 demonstra que o tema é mais do que apenas um modismo e deve permanecer em alta nos próximos anos.

A maioria das publicações selecionadas constitui-se de pesquisa teórica (de revisão de literatura) e apenas $40 \%$ apresentam pesquisas empíricas (GRÁFICO 2). Os relatos de pesquisa aplicada versam, majoritariamente, sobre protótipos e projetos-pilotos, normalmente, implementados em bibliotecas universitárias. Ou seja, são aplicações ainda em estágio inicial de desenvolvimento e ocorre no ambiente universitário, que geralmente recebe mais investimentos do que bibliotecas públicas e escolares.

Gráfico 2 - Tipo de pesquisa das publicações 


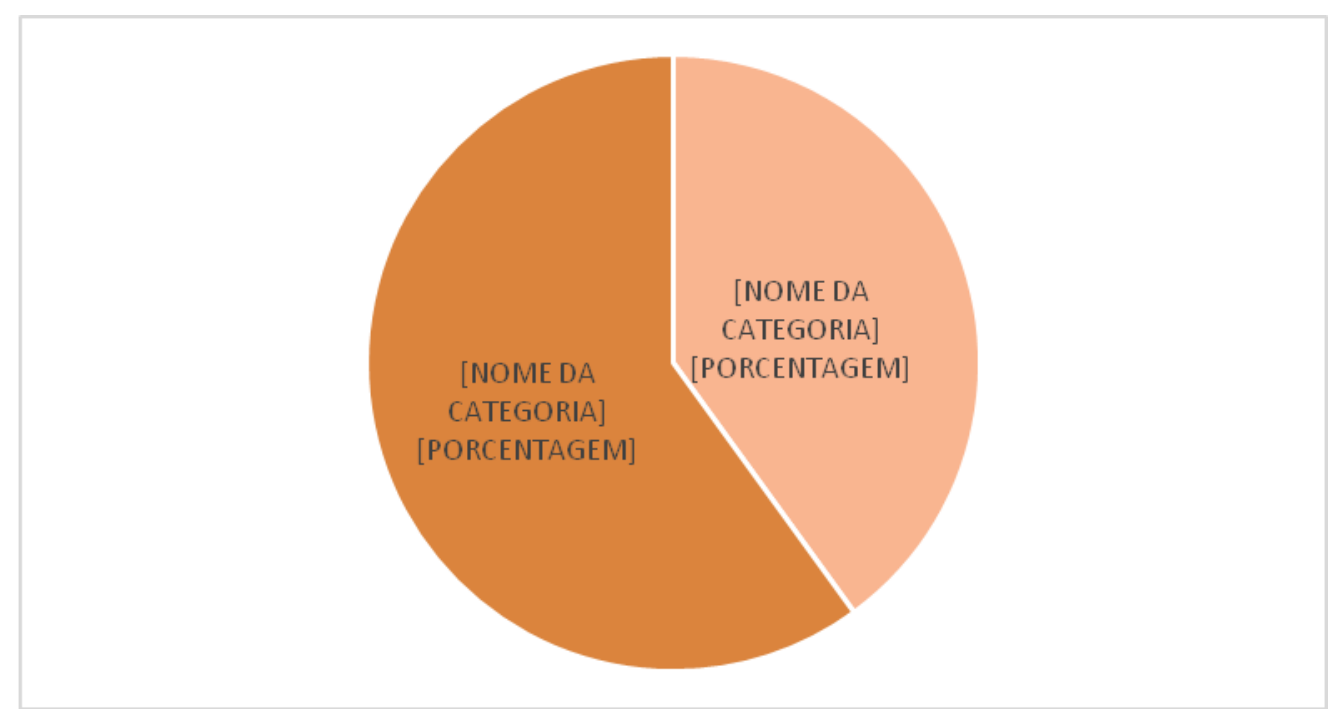

Fonte: Do autor (2019)

Mais da metade dos documentos selecionados são originários da China (GRÁFICO 3). Destaca-se também os países europeus e, por último, aparece os Estados Unidos e a Índia, com apenas um trabalho cada. A predominância chinesa demonstra que o país asiático está assumindo a dianteira na pesquisa sobre IoT em bibliotecas e pode, possivelmente, ultrapassar gigantes na área de tecnologia, como EUA e Índia.

Contudo, é importante indicar que as bases de dados Scopus e Web of Science não indexam publicações da ALA (American Library Association) e OCLC (Online Computer Library Center). Essas duas instituições americanas possuem relatórios próprios sobre a aplicação de IoT em bibliotecas (OCLC, 2015; HAHN, 2017), que não foram recuperados na busca de revisão sistemática.

Gráfico 3 - País de origem das publicações

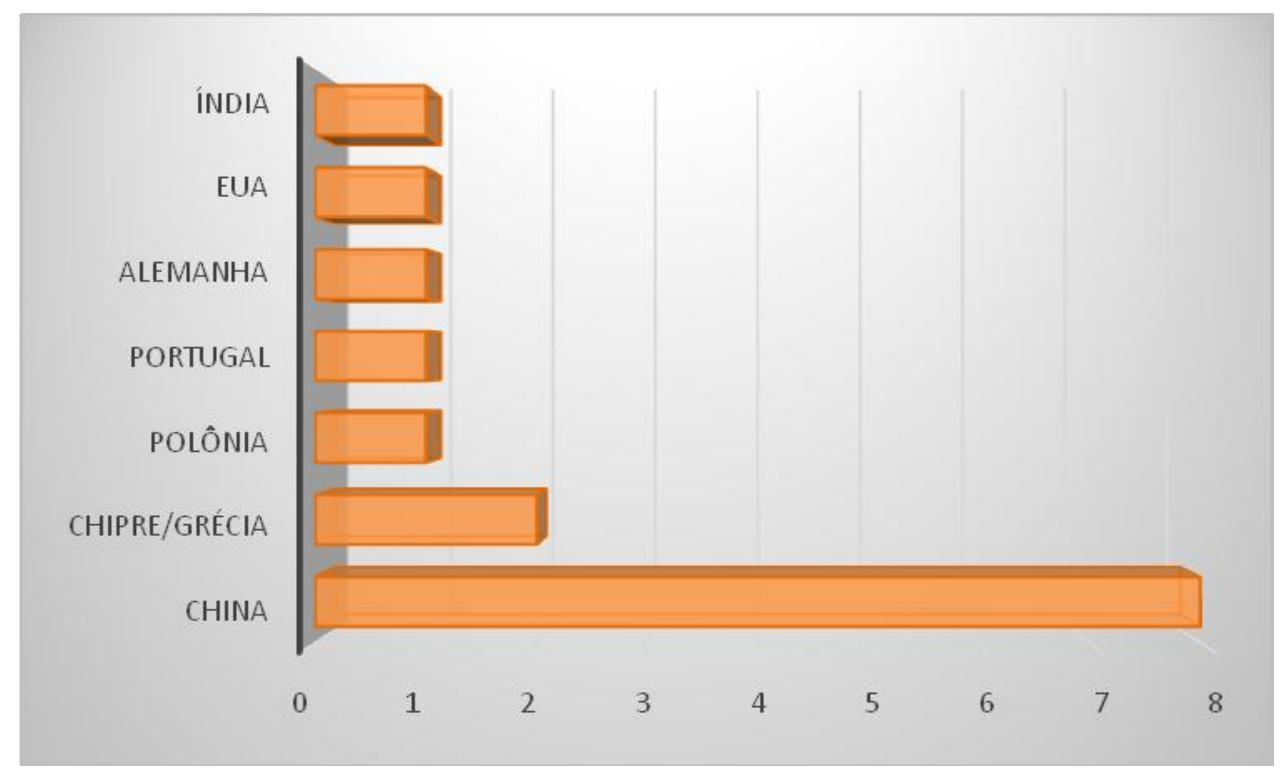


Fonte: Do autor (2019)

As bases de dados Scopus e Web of Science indicam o campo científico a que cada publicação pertence, sendo comum que um documento faça parte de mais de uma área ao mesmo tempo (GRÁFICO 4). Muitos documentos, por exemplo, pertencem tanto a área de Ciência da Computação quanto de Biblioteconomia/Ciência da Informação.

Gráfico 4 - Áreas do conhecimento com mais publicações

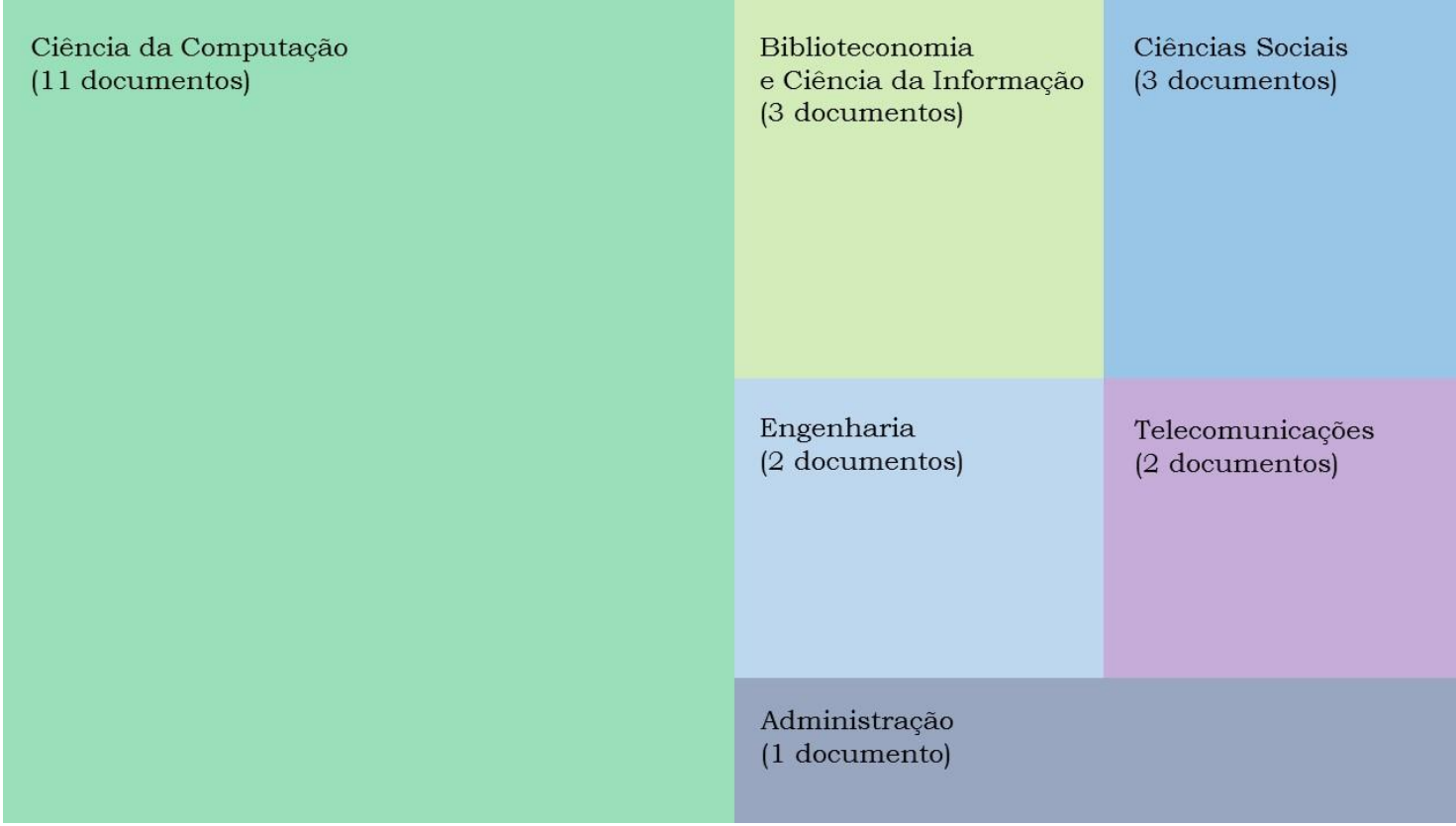

Fonte: Do autor (2019)

É possível perceber também que a Scopus faz uma classificação mais generalista das áreas científicas (Ciências Sociais), enquanto a Web of Science é mais específica (Biblioteconomia e CI). Por isso, ao fazer a análise dos documentos duplicados, que aparecem nas duas bases, optouse por utilizar a nomenclatura de áreas da Web of Science por ser mais precisa.

O campo científico da Ciência da Computação foi o que mais publicou sobre IoT em bibliotecas, com 11 documentos ao todo. A Biblioteconomia e $\mathrm{CI}$ apresentou apenas 3 documentos, contudo, por meio 
da análise das publicações classificadas pela Scopus como Ciências Sociais, infere-se que esses documentos também estão inseridos no campo da CI.

Em relação as tecnologias utilizadas, a mais citada é a identificação por rádio frequência. O RFID já é amplamente utilizado em bibliotecas para etiquetar livros e outros materiais bibliográficos em substituição aos códigos de barras, portanto pode ser mais facilmente adaptado para criar sistemas de IoT. Outras tecnologias que se destacaram na revisão sistemática foram: Magic Mirror, computação nas nuvens; sensores, como os Beacons; rede sem fio; sistema de posicionamento global (GPS).

As aplicações de IoT mais comuns em bibliotecas são voltadas para - monitoramento inteligente do ambiente (com regulagem da temperatura, humidade e luminosidade) e a gestão de acervo (em especial a localização de livros e automação dos serviços de empréstimo, devolução e inventário). Vários documentos citam também a possibilidade de oferecer serviços e produtos mais customizados para os usuários, mas não oferecem muitos detalhes técnicos ou práticos sobre como seriam esses serviços e produtos.

No monitoramento do ambiente, destacam-se o protótipo desenvolvido pela Biblioteca Universitária de Bamberg, na Alemanha,para medir a temperatura e humidade (GROSSMANN; ILLIG; MATEJKA, 2017), e o sistema de monitoramento de temperatura e eletricidade na Biblioteca Pública José Saramago, em Portugal (GALVÃO et al., 2015).Essas funcionalidades são fundamentais tanto para manter o conforto dos usuários quanto para garantir a conservação e preservação dos materiais disponibilizados pela biblioteca, pois papeis e recursos eletrônicos possuem maior durabilidade se forem armazenados em locais adequados. A regulagem automática de temperatura e das luzes pode trazer também economia nos gastos orçamentários com consumo de energia, além ser um sistema capaz de detectar desperdícios, sendo, portanto, uma iniciativa em acordo com o objetivo de criar uma sociedade mais sustentável.

Na gestão do acervo, espera-se que os livros e outros itens possam ser identificados e rastreados em tempo real dentro das bibliotecas (MARKAKIS et al., 2013; WANG; KATABI, 2013; POLYCARPOU; SAMARAS; SAHALOS, 2014; SUN; GOL, 2016). Problemas como livros perdidos ou fora das estantes serão facilmente solucionados, inventários serão realizados rapidamente, serviços de autoempréstimo e autodevolução serão disponibilizados e a segurança do acervo também aumentará, dado a possibilidade de identificar a localização de cada item a qualquer momento.

O usuário terá também maior facilidade de se locomover pelos espaços da biblioteca por meio da oferta de tour virtual guiado (GUPTA; 
SINGH, 2018) ou com o uso de mapas interativos, que mostram tanto o posicionamento do usuário quanto o dos livros. Serviços de recomendação de informação baseado nos livros emprestados, nas estantes percorridas dentro da biblioteca ou no histórico de pesquisa realizadas em outros ambientes informacionais, como a internet, serão capazes de fornecer serviços e produtos mais personalizados e customizados com as necessidades de cada usuário(HUI, 2013; NIE, 2016; GUPTA; SINGH, 2018).

Percebe-se pela revisão sistemática que a IoT poderá ser aplicada em diversas atividades desenvolvidas nas bibliotecas. Os objetivos principais dessas aplicações são facilitar as rotinas desempenhadas pelos bibliotecários, potencializar a usabilidade do espaço físico e aumentar a satisfação dos usuários.

\section{Considerações finais}

O conceito de internet das coisas ainda é muito recente e promete promover mudanças tão radicais quanto as ocorridas com a Revolução Industrial e o surgimento da internet. Conectar diretamente objetos físicos a rede mundial de computadores é uma das últimas fronteiras para inserir por completo a realidade humana no ciberespaço, podendo até mesmo trazer grandes contribuições para a criação de sistemas baseados em inteligência artificial. Espera-se com essa tecnologia que a informática seja capaz de solucionar mais rapidamente os problemas da sociedade e oferecer opções de serviços mais assertivos, precisos e que tragam mais comodidade.

Estudos sobre IoT apontam as bibliotecas como um local a ser beneficiado pela aplicação dessa tecnologia. Afinal essas unidades de informação possuem um fluxo constante de pessoas e objetos (materiais bibliográficos, entre outros).

As pesquisas sobre IoT em bibliotecas ainda são escassas e constituem-se principalmente de estudos exploratórios. A Ásia e Europa estão na dianteira na publicação de trabalhos científicos sobre o tema, ultrapassando os Estados Unidos, que possuem, normalmente, uma força muito grande tanto na área de tecnologia quanto de Biblioteconomia.

O campo do conhecimento com maior número de publicações é a Ciência da Computação, com poucas iniciativas originárias da Biblioteconomia e Ciência da Informação. Embora esse cenário seja compreensível, dado que a IoT é uma tecnologia da área de informática, é importante destacar que a Biblioteconomia deve ser uma voz atuante nas pesquisas tanto para definir as funcionalidades esperadas dos sistemas quanto para exigir a segurança dos dados coletados por sistemas de IoT, lembrando que a área é conhecida por uma ética profissional que preza pela transparência informacional e zela pela privacidade dos usuários. 
Uma das tecnologias proeminentes nos sistemas de IoT é o RFID, que já está inserido em inúmeras bibliotecas como um substituto do código de barras. Espera-se com a IoT que as funcionalidades do RFID sejam potencializadas para facilitar a rotina dentro da biblioteca e satisfazer melhor as necessidades dos usuários. $O$ fato da rádio frequência já ser uma tecnologia cotidiana dentro das bibliotecas poderá ser um facilitador na implantação de sistemas baseado na internet das coisas.

Em relação a aplicação de IoT, destacam-se pesquisas que envolvem 0 monitoramento do ambiente físico da biblioteca, o rastreamento de livros e de usuários, e serviços de recomendação.Essas funcionalidades promovem a melhor utilização dos serviços, produtos e espaço das bibliotecas.

Em conclusão, é possível identificar um interesse em aplicar a tecnologia de IoT em bibliotecas, com inúmeras possibilidades sendo aventadas. Embora o número de pesquisas práticas seja reduzido, prevalecendo estudos teóricos e exploratórios, a tendência é que novas soluções de IoT sejam testadas nas bibliotecas e sirvam de modelo para outras unidades de informação.

\section{Referências}

CAO, G.; LIANG, M.; LI, X. How to make the library smart? The conceptualization of the smart library. The Electronic Library, Bingley, v. 36, n. 5, p. 811-825, 2018. Disponível em: https://www.emeraldinsight.com/doi/abs/10.1108/EL-11-20170248?journalCode=el. Acesso em: 24 abr. 2019.

FUNCHAL, P. O que é a indústria 4.0 e o que eu tenho a ver com isso? 2017. Disponível em: https://xgb.com.br/o-que-e-industria-4-0-e-o-queeu-tenho-ver-com-isso/. Acesso em: 24 abr. 2019.

GALVÃO, J. R. et al. Energy Systems Models for Efficiency Towards Smart Cities. In: INTERNATIONAL CONFERENCE ON COMPUTER AS A TOOL, 16., Salamanca, 2015. Proceedings [...]. [New Jersey: IEEE], 2015. p. 653658. Disponível em: https://ieeexplore.ieee.org/document/7313682. Acesso em: 24 abr. 2019.

GROSSMANN, M.; ILLIG, S.; MATEJKA, C. Environmental Monitoring of Libraries with MonTreAL. In: INTERNATIONAL CONFERENCE ON THEORY AND PRACTICE OF DIGITAL LIBRARIES, 21., 2017, Thessaloniki.

Proceedings [...]. Thessaloniki: Aristotle University of Thessaloniki, 2017. p. 599-6002. Disponível em: https://link.springer.com/chapter/10.1007/978-3-319-67008-9_52.

Acesso em: 24 abr. 2019. 
GUPTA, J.; SINGH, R. Internet of Things (IoT) and Academic Libraries: a user friendly facilitator for patrons. In: INTERNATIONAL SYMPOSIUM ON EMERGING TRENDS AND TECHNOLOGIES IN LIBRARIES AND INFORMATION SERVICES, 5., 2018, Noida. Proceedings[...]. [New Jersey: IEEE], 2018. p. 71-74. Disponível em: https://ieeexplore.ieee.org/document/8485234. Acesso em: 24 abr. 2019.

HAHN, Jim. The Internet of Things: Mobile Technology and Location Services in Libraries. Library Technology Reports: Expert Guides to Library Systems and Services, v. 53, n. 1, Jan. 2017. ALA American Library Association. Disponível em:

https://journals.ala.org/index.php/Itr/issue/viewIssue/621/384. Acesso em: 24 abr. 2019.

HUI, X. Research on the Construction of Personalized Service System in the Library of Higher Vocational College Based on the Environment of Internet of Things. In: INTERNATIONAL CONFERENCE ON SCIENCE AND SOCIAL RESEARCH, 2., Beijing, 2013. Proceedings [...]. [Paris: Atlantis], 2013. p. 275-278. Disponível em: https://www.atlantispress.com/proceedings/icssr-13/7709. Acesso em: 24 abr. 2019.

LI, L. Designing and Implementation of University Library Automatic Management System Based on the Internet of Things. In: ZU, Q.; VARGAS-VERA, M.; HU, B. (Eds.) Pervasive Computing and the Networked World. New York: Springer, 2013. Joint International Conference on Pervasive Computing and Web Society, Vina del Mar, 2013. Disponível em: https://link.springer.com/chapter/10.1007/978-3-319-09265-2_25. Acesso em: 24 abr. 2019.

LIANG, X. Internet of Things and its applications in libraries: a literature review. Library Hi Tech, [Ann Arbor], 2018. Disponível em:

https://www.emeraldinsight.com/doi/abs/10.1108/LHT-01-2018-0014. Acesso em: 24 abr. 2019.

LIANG, X.; CHEN, H. The application of CPS in library management: a survey. Library Hi Tech, [Ann Arbor], 2018. Disponível em: https://www.emeraldinsight.com/doi/abs/10.1108/LHT-11-2017-0234. Acesso em: 24 abr. 2019.

LUO, Y. C. ; CAO, J. J.; QIAN, J. F. Exploration and const ruction of smart library based on RFID technology. Advanced Materials Research, Zurich, v. 765-767, p. 1743-1746, 2013. Disponível em: https://www.scientific.net/AMR.765-767.1743. Acesso em: 24 abr. 2019.

MARKAKIS, I. et al. An RFID-enabled library management system using low-SAR smart bookshelves. In: INTERNATIONAL CONFERENCE ON ELECTROMAGNETICS IN ADVANCED APPLICATIONS, Torino, 2013. 
Proceedings [...]. [New Jersey: IEEE], 2013. p. 227-230. Disponível em: https://ieeexplore.ieee.org/abstract/document/6632228/. Acesso em: 24 abr. 2019.

NIE, W. The Application of Internet of Things in the University Library. In: INTERNATIONAL CONFERENCE ON EDUCATION, MANAGEMENT, COMPUTER AND MEDICINE, 7., 2016, Shenyang. Proceedings [...]. [Paris: Atlantis], 2016. p. 24-27. (Advances in Computer Science Research, 59). Disponível em: https://www.atlantis-press.com/proceedings/emcm16/25870385. Acesso em: 24 abr. 2019.

OCLC Online Computer Library Center. The internet of things: 50 billion connected devices and objects by the year 2020. Next space, Dublin (USA), n. 24, Jan. 2015. Disponível em:

https://www.oclc.org/content/dam/oclc/publications/newsletters/nextspac e/nextspace_024.pdf. Acesso em: 24 abr. 2019.

POLYCARPOU, A. C.; SAMARAS, T.; SAHALOS, J. N. An RFID-Based Library Management System Using Smart Cabinets: A Pilot Project. In: EUROPEAN CONFERENCE ON ANTENNAS AND PROPAGATION, 8., Hague, 2014. Proceedings [...]. [Bruxelles: EurAAP], 2014. Disponível em: https://ieeexplore.ieee.org/document/6902447. Acesso em: 24 abr. 2019.

SAMPAIO, R. F.; MANCINI, M. C. Estudos de revisão sistemática: um guia para síntese criteriosa da evidência científica. Revista Brasileira de Fisioterapia, São Carlos, v. 11, n. 1, p. 83-89, fev. 2007. Disponível em: http://www.scielo.br/scielo.php?script=sci_arttext\&pid=S1413 35552007000100013. Acesso em: 24 abr. 2019.

SHIN, D.-H. A realization of pervasive computing: ubiquitous city. In: TECHNOLOGY MANAGEMENT FOR GLOBAL ECONOMIC GROWTH, 2010, Phuket, 2010. Proceedings [...]. [New Jersey: IEEE], 2010. p. 1-10. Disponível em: https://ieeexplore.ieee.org/document/5603449/. Acesso em: 24 abr. 2019.

SUN, Y.; GOU, H. A New IoT Method for Economical Library Management. In: INTERNATIONAL CONFERENCE ON WIRELESS COMMUNICATION AND NETWORK ENGINEERING, 2., Beijing, 2016. Proceedings [...]. [Lancaster: DEStech], 2016. Disponível em: http://dpiproceedings.com/index.php/dtcse/article/view/5148/4774. Acesso em: 24 abr. 2019.

WANG, J.; KATABI, D. Dude, Where's My Card? RFID Positioning That Works with Multipath and Non-Line of Sight. In: ACM SIGCOMM CONFERENCE, Hong Kong, 2013. Proceedings [...]. New York: ACM, 2013. p. 51-62. Disponível em: 
http://groups.csail.mit.edu/netmit/6.888/www/papers/pinit.pdf. Acesso em: 24 abr. 2019.

WÓJCIK, M. Internet of Things: potential for libraries. Library Hi Tech, [Ann Arbor], v. 34, n. 2, p. 404-420, 2016. Disponível em: https://www.emeraldinsight.com/doi/abs/10.1108/LHT-10-2015-0100. Acesso em: 24 abr. 2019. 